CASE REPORT

\title{
Myocarditis as a prognostic indicator in systemic lupus erythematosus
}

\author{
S Busteed, P Sparrow, C Molloy, M G Molloy
}

Postgrad Med J 2004;80:366-367. doi: 10.1136/pgmj.2003.012450

Lupus myocarditis is a life threatening complication of systemic lupus erythematosus (SLE). A case of left ventricular failure secondary to myocarditis occurring in a patient with SLE is reported. Despite resolution of the cardiac failure with pulsed cyclophosphamide and steroids, she eventually died of non-cardiac complications 18 months later. The literature is also reviewed.

upus myocarditis is a rare but potentially fatal complication of systemic lupus erythematosus (SLE). The relative rarity of the condition excludes the feasibility of carrying out controlled trials on optimal treatment and management is based on anecdotal reports. There is also a paucity of information available on the long term outcome of patients who initially respond to treatment. Here, we report the initial course and outcome in a patient with SLE who developed left ventricular failure secondary to myocarditis.

\section{CASE REPORT}

A 23 year old woman with SLE presented acutely with a 48 hour history of dyspnoea, pedal oedema, and orthopnoea. She also complained of central chest pain exacerbated by coughing and of blurred vision in the left eye. She was diagnosed with SLE seven years previously on the basis of a malar rash, arthralgia, and positive antinuclear antibody and double stranded DNA antibodies but her initial disease course had been relatively quiescent, requiring only antimalarial treatment for a number of years. She had been discharged five days previously after a lengthy admission with

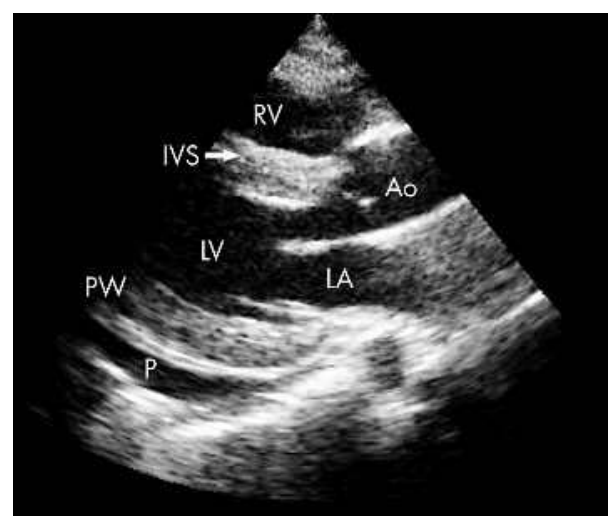

Figure 1 Transthoracic echocardiogram parasternal long axis image. Note the presence of a small pericardial effusion with a grossly thickened echogenic septum and posterior wall of the left ventricle. The mitral and aortic valves were normal (Ao, aorta; IVS, interventricular septum; LA, left atrium; LV, left ventricle; $\mathrm{P}$, pericardial effusion; PW, posterior wall; $\mathrm{RV}$, right ventricle).

Haemophilus influenzae pneumonia, neutropenia, and hepatitis. On examination, she was tachypnoeic with a respiratory rate of 32 breaths per minute, had a raised blood pressure of 140/105 mm Hg, and was tachycardic with a heart rate of 115 beats per minute. She also had a low grade pyrexia of $37.8^{\circ} \mathrm{C}$. Cardiovascular examination revealed a raised jugular venous pressure with a marked S3 and bibasal crepitations on auscultation. There was pronounced sacral and pedal oedema. Cotton wool spots were noted on the left retina. An electrocardiogram showed sinus rhythm, normal axis, and T-wave flattening from V2 to V4. A chest radiograph revealed bibasal and right midzonal shadowing. She was found to be hypoxic with a respiratory alkalosis and normal renal function (laboratory investigations are outlined in table 1). Echocardiography demonstrated moderate global left ventricular systolic impairment with a thickened echogenic ventricular wall and an estimated ejection fraction of $40 \%$ (see fig 1). A small pericardial effusion was also evident. The findings demonstrated considerable deterioration compared with a previous echocardiogram carried out one month before. A diagnosis of acute myocarditis secondary to SLE was made, based on the clinical findings and investigations. She was started on pulsed intravenous methylprednisolone ( $1 \mathrm{~g}$ daily for three days) and was given a $1 \mathrm{~g}$ intravenous infusion of cyclophosphamide. Her heart failure was treated with intravenous frusemide, digoxin, and enalapril.

Two days into her admission, symptoms of pericarditis with severe chest pain persisted and a repeat electrocardiogram showed new lateral T-wave inversion in leads V4-V6. Creatine kinase levels remained normal. Blood pressure remained raised (165/115) and a gallop rhythm with a marked S3 and S4 was noted. However within five days of the start of treatment, the clinical signs of heart failure had resolved and the patient was clinically much improved. Repeat echocardiography confirmed that the ejection fraction had improved to $55 \%$, although the myocardium remained echogenic with a $1 \mathrm{~cm}$ pericardial effusion. A second pulse of cyclophosphamide was administered a week after admission. Serology for anti-Ro, anti-La, and antiribonucleoprotein antibodies was negative and titres for coxsackie, adenovirus,

Table 1 Laboratory investigations

\begin{tabular}{lll}
\hline Investigation & Value & Normal range \\
\hline Oxygen tension (kPa) & 8.5 & $11-14$ \\
$\mathrm{pH}$ & 7.49 & $7.35-7.45$ \\
Haemoglobin (g/l) & 94 & $120-160$ \\
Creatine kinase (IU/l) & 22.5 & $40-180$ \\
Albumin (g/l) & 25 & $36-44$ \\
dsDNA (IU/l) & $>200$ & $<35$ \\
C3 (g/l) & 0.29 & $0.75-1.65$ \\
Erythrocyte sedimentation & 47 & $<20$ \\
rate (mm/hour) & & \\
C-reactive protein (mg/l) & 6.2 & $<10$ \\
\hline
\end{tabular}


and Coxiella burnetti were also negative. A pre-discharge echocardiogram at 19 days showed complete resolution of both myocardial changes and pericardial effusion with improvement of systolic function and normal diastolic function. Four further pulses of cyclophosphamide were given intravenously at fortnightly intervals giving a $6 \mathrm{~g}$ cumulative dose.

Despite remission of the cardiac signs and symptoms, her systemic disease remained active and she developed lupus nephritis, which necessitated the use of mycophenolate mofetil. Twenty months after the episode of myocarditis, she was readmitted with seizures secondary to cerebral vasculitis and renal failure and required transfer to intensive care. She died two months later as a consequence of systemic involvement with multiorgan failure. Of note, myocardial involvement was not detected on follow up echocardiography, although there was evidence of a small residual pericardial effusion. A postmortem examination was not carried out.

\section{DISCUSSION}

Myocardial involvement is not uncommon in SLE. Evidence of myocarditis can be found at postmortem examination in approximately $40 \%$ of cases ${ }^{1}$ and also in echocardiographic studies with about $6 \%$ of SLE patients showing global hypokinesia. ${ }^{2}$ A prospective echocardiographic study of 70 patients found myocardial abnormalities in 14 cases $(20 \%)$ but only one patient had symptoms. ${ }^{3}$ However, the development of symptomatic left ventricular failure is rare. Myocarditis accounted for only one out of 43 deaths in a series of 544 patients. ${ }^{4}$ Myocarditis may manifest itself as an acute illness or have a chronic course with the development of a cardiomyopathy due to small vessel vasculitis and thrombosis. ${ }^{1}$ Myocarditis may rarely be the initial presentation of SLE. ${ }^{5}$

Myocarditis can be diagnosed on clinical grounds supported by laboratory findings (leukocytosis, raised erythrocyte sedimentation rate, raised creatine kinase and troponins $\mathrm{T}$ and I) and transthoracic echocardiography. Endomyocardial biopsy can give definitive histology but is limited by a lack of sensitivity and specificity and histological findings should be taken in the context of the clinical picture. Electrocardiographic findings such as dysrhythmias or heart block may be seen. There has been speculation about the association of lupus myocarditis with the presence of anti-Ro and antinuclear ribonucleoprotein antibodies. ${ }^{78}$ Antimyosin scintigraphy has also been used in the diagnosis of myocarditis. ${ }^{9}$

The treatment of lupus myocarditis has in general, been empirical. Steroids have been the mainstay of treatment either orally or as intravenous pulses. ${ }^{5}$ Cyclophosphamide has been used with success ${ }^{10}{ }^{11}$ and there are reports of improvement with high dose intravenous immunoglobulin ${ }^{12}$ and plasmapheresis. ${ }^{13}$ Extracorporeal membrane oxygenation ${ }^{14}$ has been used successfully in lupus myocarditis associated with cardiogenic shock. There is little information available on the long term outcome in patients with SLE who develop myocarditis and are successfully treated. This case suggests that lupus related myocarditis carries a poor prognosis in terms of long term survival despite initial clinical remission.

\section{Authors' affiliations}

S Busteed, Department of Rheumatology, Royal Liverpool University Hospital, Liverpool, UK

P Sparrow, Department of Cardiology, Cork University Hospital, Wilton, Cork

C Molloy, M G Molloy, Department of Rheumatology, Cork University Hospital, Wilton, Cork

Correspondence to: Dr S Busteed, Department of Rheumatology, Royal Liverpool University Hospital, Prescot Street, Liverpool L7 8XP, UK; sandra_busteed@hotmail.com

Submitted 12 July 2003

Accepted 19 November 2003

\section{REFERENCES}

1 Doherty NE, Siegel RJ. Cardiovascular manifestations of systemic lupus erythematosus. Am Heart J 1985; 110:1257-65.

2 Wijetunga $M$, Rockson S. Myocarditis in systemic lupus erythematosus. Am J Med 2002;1 13:419-23.

3 Cervera R, Font J, Pare C, et al. Cardiac disease in systemic lupus erythematosus: prospective study of 70 patients. Ann Rheum Dis 1992;51:156-9.

$4 \mathrm{Kim}$ WU, Min JK, Lee SH, et al. Causes of death in Korean patients with systemic lupus erythematosus: a single centre retrospective study. Clin Exp Rheumatol 1999:17:539-45.

5 Frustaci A, Gentiloni N, Caldarulo M. Acute myocarditis and left ventricular aneurysm as presentations of systemic lupus erythematosus. Chest 1996;109:282-4

6 Feldman AM, McNamara D. Myocarditis. N Engl J Med 2000;343:1388-98.

7 Logar D, Kveder T, Rozman B, et al. Possible association between anti-Ro antibodies and myocarditis or cardiac conduction defects in adults with systemic lupus erythematosus. Ann Rheum Dis 1990;49:627-9.

8 Swaak AJ, Huysen V, Smeenk RJ. Antinuclear antibodies in routine analysis: the relevance of putative clinical associations. Ann Rheum Dis 1993;52:110-4.

9 Morguet AJ, Sandrock D, Stille-Siegener M, et al. Indium-111-antimyosin Fab imaging to demonstrate myocardial involvement in systemic lupus erythematosus. J Nucl Med 1995;36:1432-5.

10 Ueda T. Echocardiographic observation of acute myocarditis with systemic lupus erythematosus. Jpn Circ J 2000;64:144-6.

11 Hammann C, Genton CY, Delabays A, et al. Myocarditis of mixed connective tissue disease: favourable outcome after intravenous pulsed cyclophosphamide. Clin Rheumatol 1999;18:85-7.

12 Sherer $Y$, Levy Y, Shoenfeld Y. Marked improvement of severe cardiac dysfunction after one course of intravenous immunoglobulin in a patient with systemic lupus erythematosus. Clin Rheumatol 1999;18:238-40.

13 Tamburino C, Fiore CE, Foti R, et al. Endomyocardial biopsy in diagnosis and management of cardiovascular manifestations of systemic lupus erythematosus (SLE). Clin Rheumatol 1989;8:108-12.

14 Leung MC, Harper RW, Boxall J. Extracorporeal membrane oxygenation in fulminant myocarditis complicating systemic lupus erythematosus. Med J Aust 2002; 176:374-5. 